\title{
Investigation of the Leaky-Wave Characteristics of a Cylindrical Dielectric Rod Using the Coefficient Matrix of the System of Characteristic Equations and Davidenko's Method
}

\author{
Ersoy Kelebekler ${ }^{*}$
}

\begin{abstract}
An open dielectric waveguide includes both guided propagating modes within the waveguide and radiating and evanescent modes that propagate transversely outside the waveguide. In this study, the leaky-wave characteristics of a cylindrical dielectric rod are investigated using the coefficient matrix of the system of characteristic equations and Davidenko's method. Using the coefficient matrix of the system of characteristic equations simplified the derivative(s) required to use Davidenko's method. The results obtained for a 10-mm radius structure with a relative dielectric constant of 4 showed that a second antenna mode region existed for higher-order hybrid HE modes and lower-order transverse magnetic (TM) modes. However, it did not exist for transverse electric (TE) modes. Additionally, the hybrid HE modes had larger leaky-wave spectrum and antenna mode regions than both the TE and TM modes. This demonstrates that for antenna applications, hybrid HE modes supply a wider frequency spectrum.
\end{abstract}

Key Words: Cylindrical Dielectric Rod, Davidenko's Method, Hybrid Electromagnetic Modes, Leaky Waves.

\section{INTRODUCTION}

The electromagnetic theory states that the scattering of the wave from a dielectric cylinder has both non-radiative guided modes and radiative leaky modes with discrete eigenvector solutions [1]. A leaky wave radiates along an interface, perpetually attenuating its energy by propagation [2, 3]. Leaky-wave modes in optical communication also refer to natural optical modes with propagating waves outside the structure that continuously lose energy through radiation [4]. Wave leakage from waveguide structures depends on many factors, such as the material filling the structure, physical geometry, guided mode, and operating frequency. A leaky wave radiating or leaking along an open waveguide structure allows for a frequency-dependent beam-scanning structure or leaky antenna structure, which allows energy to radiate to remote areas [5]. Over the last few decades, the advantages of leaky-wave structures, such as their simple structure, easy fabrication, high directivity, fairly narrow pattern bandwidth, cost efficiency, and applicability from millimeter-wave frequency to optical frequency, have made them popular research subjects [6-28].

Manuscript received September 1, 2020 ; Revised November 28, 2020 ; Accepted January 28, 2021. (ID No. 20200901-132J)

Department of Electricity and Energy, Uzunciftlik Nuh Cimento VHS, Kocaeli University, Kocaeli, Turkey.

"Corresponding Author: Ersoy Kelebekler (e-mail: ersoy@kocaeli.edu.tr)

This is an Open-Access article distributed under the terms of the Creative Commons Attribution Non-Commercial License (http://creativecommons.org/licenses/by-nc/4.0) which permits unrestricted non-commercial use, distribution, and reproduction in any medium, provided the original work is properly cited.

(c) Copyright The Korean Institute of Electromagnetic Engineering and Science. 
In a leaky-wave open dielectric structure, the energy traveling along the interface leaks through the open waveguide structure, and the wave is then reflected in the interior dielectric. The leaked energy then radiates to some distance from the open waveguide structure. This phenomenon can be mathematically modeled by complex roots of the propagation constant or the eigenvalue equation. These complex solutions, called leaky modes, are not members of a complete set of orthogonal basis functions [29]. These complex solutions are nonspectral and also called improper modes because of their unphysical characteristics, in which the transverse components of the leaky mode increase exponentially while moving in the transverse direction. Although this is physically impossible, as it means violating the boundary condition at infinity, leaky waves can be physically measured and used in many electromagnetic applications. However, since these waves are mathematically improper, they can only exist within a constrained region in the form of a wedge, where the field remains finite [3032].

Open dielectric guide structures are often used as leaky-wave antennas or waveguides in integrated circuits (ICs). In the optical communication spectrum, an open waveguide structure acts as a leaky antenna and waveguide at different frequency regions. This situation requires careful study to determine the operation region for practical applications. An open dielectric guiding structure used as an antenna operates in the high radiation efficiency frequency region. In contrast, ICs work in the frequency region where the leakage effect must be eliminated as much as possible. As a result, the aim of a leakage investigation for antenna applications is slightly different than for ICs. In the first case, leakage is very desirable; in the second case, leakage is undesirable and should be suppressed [33]. The dispersion characteristics of the structure determine the boundaries of the two operation regions. In other words, frequency-dependent modal behavior classifies the relevant areas. In the literature, the propagation regions for open waveguide structures are divided into four subregions with decreasing frequency as follows [33-37]:

1) The bound-mode region, where the mode propagates unattenuated toward the propagation direction, and the solution for guidance along an open structure is spectral with a pure real propagation constant (no attenuation constant).

2) The surface-wave region, where the guided mode leaks power along the surface of the open structure while propagating toward the propagation direction, and the solution is spectral and complex with a small attenuation constant.

3) The antenna mode region, where the guided mode mostly leaks power into the space wave and surface wave, and the solution is nonspectral and complex with little attenuation constant.

4) The reactive mode region, where the guided mode attenuates due to the reflection of energy back to the feed line, and the solution is nonspectral and complex, with a significant attenuation constant.

In the literature, leaky-wave modes for open waveguide structures with different geometries have been studied with the aid of various analytical methods: an open microstrip line of an arbitrary cross-section, with the integral equation technique correlatively with the method of moments [38]; a partial dielectric-loaded open waveguide by examining the behavior of a complex propagation constant [31]; a microstrip transmission line with a rigorous spectral-domain integral equation formulation [39]; a microstrip leaky-wave antenna with a full-wave spectral-domain integral equation method [34, 35]; the analytical expression of a simple cavity model [40]; and an open dielectric tube waveguide loaded with plasma using the analytical solution of the structure and the Muller's complex root search algorithm [37]. The leaky-wave characteristics of a circular dielectric rod for transverse magnetic (TM) and transverse electric (TE) modes have been studied using the dispersion relations of the structure and Davidenko's method $[32,36,41]$. The frequency spectrum for the structure has been divided into five subregions: guided mode, nonphysical mode, reactive mode, spectral gap, and antenna mode. Our study aimed to obtain the leaky-mode characteristics of hybrid electromagnetic (HEM) modes for a cylindrical dielectric rod with the aid of $\mathrm{Da}^{-}$ videnko's method using approximations different from methods published in the literature.

Due to the metallic boundary, the guided modes for TE $\left(\mathrm{E}_{\mathrm{z}}=\right.$ $0)$, TM $\left(\mathrm{H}_{\mathrm{z}}=0\right)$, and TEM $\left(\mathrm{E}_{\mathrm{z}}\right.$ and $\left.\mathrm{H}_{\mathrm{z}}=0\right)$ exist in metallic waveguides. An open waveguide structure has HEM modes as well as TM and TE modes due to the nonconducting boundary condition because all magnetic and electrical field components exist both inside and outside the boundary. The azimuthal variation for HEM modes is not zero $(m \geq 1)$ [42-44]. The HEM modes are designated as $\mathrm{HE}$ and $\mathrm{EH}$ modes, depending on the relative contributions of the longitudinal magnetic field and the longitudinal electric field [45-47]. In this study, the leaky-wave characteristics of the modes for a cylindrical dielectric rod were obtained using the coefficient matrix of the system of characteristic equations of the structure and Davidenko's method. Davidenko's method is a robust technique used to compute the complex roots of analytical functions. Davidenko's method has been used for electromagnetic problems in various studies [32, 36, 41, 4854]. Kim et al. studied the leaky-wave characteristics of TM and TE modes for cylindrical dielectric rods using closed dispersion equations of the structure and Davidenko's method [32, 36, 41]. Davidenko's method requires the derivative of the closed dispersion equation with respect to the propagation constant. Because it is very difficult to differentiate an implicit function with interdependent variables, Kim and his colleagues [32, 36, 41] numerically obtained the derivative of the equation with the aid of MATHEMATICA 4.0. Unlike the study of Kim et al. [32, 36, 
41], in this study, the coefficient matrix of the system of characteristic equations was used instead of the dispersion equation of the structure. Thus, the auxiliary software programs required for the derivative of the equation were removed, and a complete analytical solution set is presented. This approach allows analytical solutions of problems with more complex solution sets to be obtained, such as the hybrid modes of a cylindrical dielectric rod and the electromagnetic wave in an anisotropic medium using $\mathrm{Da}$ videnko's method.

This study's results for the TM and TE leaky modes were compared to results in the literature. The obtained leaky-wave characteristics of the HEM modes of the structure represent an original contribution to the literature. In the next two sections, first Davidenko's method and then its application to a cylindrical dielectric rod are explained. The numerical results obtained for the TE, TM and HE modes and an interpretation of the results are given in Section IV. The paper ends with a conclusion.

\section{DAVIDENKO'S METHOD}

Davidenko's Newton-based method is used to transform the $\mathrm{n}$-dimension system of nonlinear algebraic equations $(n \geq 2)$ into a set of $\mathrm{n}$ first-order ordinary differential equations (ODEs) in a scalar dummy variable $t[48,51,55]$. The method is a robust technique used to compute the complex roots of transcendental equations. While Davidenko's method is insensitive to initial guesses, traditional, complex root-finding algorithms, such as the Newton-Raphson and Muller's methods, need to approximate an initial guess. Additionally, the Davidenko's method has a higher speed of convergence than conventional methods. Davidenko's method is utilized as a scalar dummy variable when transforming with the Jacobian matrix, a system of nonlinear algebraic equations, to a set of $n$ first-order ODEs. As the dummy variable $t$ approaches infinity, the dispersion equation steady-state solution for a large dummy variable yields the complex roots of the system $[32,36,48,53]$. The formal expression of Davidenko's method for the $n$-dimensional case of algebraic functions with $n$-unknowns, $\boldsymbol{f}(x)=\mathbf{0}$, is presented in the following equation:

$$
\frac{d x}{d t}=-(\boldsymbol{J})^{-1} \boldsymbol{f}(\boldsymbol{x}),
$$

where $d t$ corresponds to the increment of the dummy variable $t$ and $J$ is the Jacobian matrix for $n$ nonlinear algebraic equations set in $n$ unknowns.

In this study, Davidenko's method was used to compute the complex propagation constant corresponding to the complex roots of the coefficient matrix of the system of characteristic equations of the open cylindrical waveguide. The implicit dispersion equation of the structure is as follows:

$$
F(\omega ; \gamma)=0,
$$

where $\omega$ is the angular frequency and $\gamma$ is the propagation constant. The normalized propagation constant is defined as follows:

$$
\bar{\gamma}=\frac{\gamma}{k_{0}}=\frac{\beta-j \alpha}{k_{0}}=\bar{\beta}-j \bar{\alpha},
$$

where $\beta, \alpha$, and $k_{0}$ are the phase constant, the attenuation constant, and the free space wavenumber, respectively. For Eq. (2), both the real and imaginary parts of the complex number have to be zero; therefore, we can write the real and imaginary parts of Eq. (2) as two nonlinear equations as follows:

$$
\begin{aligned}
& \operatorname{Re}[F(\omega ; \gamma)] \equiv G(\omega ; \alpha, \beta)=0 \\
& \operatorname{Im}[F(\omega ; \gamma)] \equiv H(\omega ; \alpha, \beta)=0 .
\end{aligned}
$$

The dispersion equation, given in Eq. (2), is a complex analytic function on the complex propagation plane except at a finite number of points. Analytic functions are satisfied by CauchyRiemann equations, and the expressions given above can be written as follows:

$$
\begin{aligned}
G_{\alpha} & =H_{\beta} \\
G_{\beta} & =-H_{\alpha},
\end{aligned}
$$

where the subscripts represent the corresponding derivatives. The Jacobian matrix of the equations system given in Eq. (4) can be obtained as follows:

$$
\boldsymbol{J}=\left[\begin{array}{ll}
G_{\alpha} & G_{\beta} \\
H_{\alpha} & H_{\beta}
\end{array}\right]
$$

where, from Eq. (5),

$$
\boldsymbol{J}=\left[\begin{array}{cc}
G_{\alpha} & G_{\beta} \\
-G_{\beta} & G_{\alpha}
\end{array}\right],
$$

and from the properties of the analytic function,

$$
\frac{\partial F}{\partial \gamma} \equiv F_{\gamma}=G_{\alpha}+j H_{\alpha}=G_{\alpha}-j G_{\beta},
$$

where

$$
\begin{aligned}
G_{\alpha} & =\operatorname{Re}\left[F_{\gamma}\right] \\
G_{\beta} & =-\operatorname{Im}\left[F_{\gamma}\right] .
\end{aligned}
$$

The determinant of the Jacobian matrix can be obtained using Eqs. (7) and (9) as follows:

$$
\operatorname{det} \boldsymbol{J}=G_{\alpha}^{2}+G_{\beta}^{2}=\left|F_{\gamma}\right|^{2} .
$$

From the obtained expression and using the definition of the inverse matrix, the inverse Jacobian matrix given in Eq. (1) for the dispersion equation can be achieved, as in Eq. (11) as follows:

$$
\boldsymbol{J}^{-1}=\frac{1}{\operatorname{det} \boldsymbol{J}} \text { adj } \boldsymbol{J}=\frac{1}{G_{\alpha}^{2}+G_{\beta}^{2}}\left[\begin{array}{cc}
G_{\alpha} & G_{\beta} \\
-G_{\beta} & G_{\alpha}
\end{array}\right]
$$




$$
=\frac{1}{\left|F_{\gamma}\right|^{2}}\left[\begin{array}{cc}
\operatorname{Re}\left[F_{\gamma}\right] & \operatorname{Im}\left[F_{\gamma}\right] \\
-\operatorname{Im}\left[F_{\gamma}\right] & \operatorname{Re}\left[F_{\gamma}\right]
\end{array}\right],
$$

where adj corresponds to the adjugate of the matrix. The formal expression of Davidenko's method is eventually obtained as the following expressions:

$$
\begin{gathered}
\frac{d}{d t}\left[\begin{array}{l}
\alpha \\
\beta
\end{array}\right]=-\boldsymbol{J}^{-1}\left[\begin{array}{l}
G \\
H
\end{array}\right] \\
=-\frac{1}{\left|F_{\gamma}\right|^{2}}\left[\begin{array}{cc}
\operatorname{Re}\left[F_{\gamma}\right] & \operatorname{Im}\left[F_{\gamma}\right] \\
-\operatorname{Im}\left[F_{\gamma}\right] & \operatorname{Re}\left[F_{\gamma}\right]
\end{array}\right]\left[\begin{array}{l}
\operatorname{Re}[F] \\
\operatorname{Im}[F]
\end{array}\right]
\end{gathered}
$$

and

$$
\begin{gathered}
\frac{d \alpha}{d t}=-\frac{1}{\left|F_{\gamma}\right|^{2}}\left(\operatorname{Re}\left[F_{\gamma}\right] \operatorname{Re}[F]+\operatorname{Im}\left[F_{\gamma}\right] \operatorname{Im}[F]\right) \\
\frac{d \beta}{d t}=\frac{1}{\left|F_{\gamma}\right|^{2}}\left(\operatorname{Im}\left[F_{\gamma}\right] \operatorname{Re}[F]-\operatorname{Re}\left[F_{\gamma}\right] \operatorname{Im}[F]\right) .
\end{gathered}
$$

Consequently, the dispersion equation in the form of $F(\omega ; \gamma)=$ 0 is transformed into a system of two coupled first-order ODEs by Davidenko's method. The solution of the ODEs, given in Eqs. (13) and (14) for a steady-state solution for a large dummy variable, $t$ gives us the complex roots of the propagation constant $\gamma$ for a given frequency $\omega$ to the desired precision. The method evaluates the function value $F$ and its derivative with respect to the propagation constant $F_{\gamma}$ at each iteration. The derivative of an implicit function can be complicated depending on the complexity of the function and the interdependence of the variables. As mentioned, Kim and his colleagues [32, 36, 41] used an auxiliary program, MATHEMATICA 4.0, to calculate the derivative of the implicit dispersion equations for the TM and TE modes of a cylindrical dielectric rod. Unlike other studies, in the current study, an approach using the coefficient matrix of the system of characteristic equations instead of the implicit dispersion relation was used to reduce the derivative complexity and eliminate auxiliary program dependency. Details of the approach are given in the next section.

\section{APPLICATION OF DAVIDENKO'S METHOD TO A CYLINDRICAL DIELECTRIC ROD}

Davidenko's method is used to obtain complex roots of analytical functions, and the method requires the analytic expression of the function and its derivative. The derivative of an implicit function can be complicated depending on the complexity of the function and the interdependence of the variables, especially for structures with complex dispersion relationships. While utilizing an auxiliary mathematical program can be helpful, it can detract from the analytical solution and weaken the authority over the problem. Therefore, in this study, an approach was offered to facilitate the complex derivative expressions required by Davidenko's method. The approach referenced the coefficient matrix of the system of characteristic equations of the structure, the prerequisite step to achieving the implicit dispersion equation. Characteristic equations were obtained from Maxwell's equations using medium parameters, such as permittivity and permeability, boundary conditions, and continuity conditions. The zeros of the determination of the coefficient matrix of the system of characteristic equations correspond to the zeros of the implicit dispersion equation. In this case, this can be expressed as follows, if $M$ is the coefficient matrix of the system of characteristic equations:

$$
F(\omega ; \gamma)=\operatorname{det}(\boldsymbol{M})=0 .
$$

As is known, Davidenko's method requires the derivative of the function. In our approximation, it was obtained from the definition of the derivative of the determinant as follows:

$$
\frac{d F(\omega ; \gamma)}{d \gamma}=F_{\gamma}=\frac{d \operatorname{det}(\boldsymbol{M})}{d \gamma}=\operatorname{tr}\left(\operatorname{adj}(\boldsymbol{M}) \frac{d \boldsymbol{M}}{d \gamma}\right),
$$

where $t r$ stands for the trace of the matrix and adj is the adjugate of the matrix, as given in the previous section. From the definition of the inverse matrix, the derivative expression can be written as follows:

$$
F_{\gamma}=\operatorname{tr}\left(\operatorname{det}(\boldsymbol{M}) \boldsymbol{M}^{-1} \frac{d \boldsymbol{M}}{d \gamma}\right) .
$$

The derivative of a matrix with respect to a variable is obtained by the derivative of each element of the matrix with respect to the corresponding variable. Consequently, the Davidenko expressions are obtained as follows:

$$
\begin{gathered}
\frac{d \alpha}{d t}= \\
-\frac{1}{\left|\operatorname{tr}\left(\operatorname{det}(\boldsymbol{M}) \boldsymbol{M}^{-1} \frac{d \boldsymbol{M}}{d \gamma}\right)\right|^{2}}\left\{\operatorname{Re}\left[\operatorname{tr}\left(\operatorname{det}(\boldsymbol{M}) \boldsymbol{M}^{-1} \frac{d \boldsymbol{M}}{d \gamma}\right)\right] \operatorname{Re}[\operatorname{det}(\boldsymbol{M})]\right. \\
\left.+\operatorname{Im}\left[\operatorname{tr}\left(\operatorname{det}(\boldsymbol{M}) \boldsymbol{M}^{-1} \frac{d \boldsymbol{M}}{d \gamma}\right)\right] \operatorname{Im}[\operatorname{det}(\boldsymbol{M})]\right\} \\
\frac{d \beta}{d t}= \\
\frac{1}{\left|\operatorname{tr}\left(\operatorname{det}(\boldsymbol{M}) \boldsymbol{M}^{-1} \frac{d \boldsymbol{M}}{d \gamma}\right)\right|^{2}}\left\{\operatorname{Im}\left[\operatorname{tr}\left(\operatorname{det}(\boldsymbol{M}) \boldsymbol{M}^{-1} \frac{d \boldsymbol{M}}{d \gamma}\right)\right] \operatorname{Re}[\operatorname{det}(\boldsymbol{M})]\right. \\
\left.-\operatorname{Re}\left[\operatorname{tr}\left(\operatorname{det}(\boldsymbol{M}) \boldsymbol{M}^{-1} \frac{d \boldsymbol{M}}{d \gamma}\right)\right] \operatorname{Im}[\operatorname{det}(\boldsymbol{M})]\right\}
\end{gathered}
$$

Finding the derivative of the elements of the matrix is more straightforward than finding the derivative of the complex implicit expression of a structure, where many parameters depend on each other and the derivative variable. This is because the implicit expression is derived from the coefficient matrix of the system of characteristic equations by expressing the equations in terms of each other. The simplification of the derivative allows Daviden- 
ko's method to be applied to more complex structures. Also, a complete set of analytical solutions can be obtained without the need for auxiliary software programs. In the literature, the TM and TE leaky modes of a structure have been determined by $\mathrm{Da}-$ videnko's method and implicit dispersion relations [32, 36, 41], and the implicit dispersion relationships for TE, TM, and HEM modes have been given in related studies. However, only the leaky modes for TE and TM modes are obtained using Davidenko's method, and it has been proposed that hybrid leaky modes should be studied [32]. In our study, the TM and TE leaky modes of the structure were obtained by the presented approach based on the coefficient matrix of the system of characteristic equations and compared with the results in the literature. We also obtained and present the HEM leaky modes. The numerical results for the structures were obtained using MATLAB, the most widely used mathematical computational program. The guided modes, corresponding to the real roots of the implicit dispersion equation or zeros of the determination of the coefficient matrix of the system of characteristic equations for the pure real propagation constant $(\alpha=0)$ were computed using the bisection method. Leaky-wave modes corresponding to the zeros of the determination of the coefficient matrix of the system of characteristic equations for the complex propagation constant were computed using Davidenko's method.

\section{NUMERICAL RESULTS AND DISCUSSION}

Determining the operation region of an open waveguide structure is essential for the performance of antenna and ICs because it can be used as both a leaky wave antenna and a guidance structure. While an antenna requires high leakage, sometimes special efforts are necessary to suppress this to prevent spurious performance and crosstalk $[56,57]$. In this part of the study, the leaky wave modes of the cylindrical dielectric rod were investigated by Davidenko's method using the approach given in the previous section, and the operation regions for the guided mode, nonphysical mode, reactive mode, antenna mode, and spectral gap regions were computed. For an open waveguide structure, it is known that the guided mode is propagated unattenuated above the cutoff, and it leaks energy transversely into both space and surface waves in a narrow frequency region. Also, it is attenuated due to losing energy to the surface wave and reflecting it back to the feed line. The leaky wave region below the cutoff, where the mode is attenuated due to losing energy into both the space wave and surface wave, has been previously studied as the radiation region [30,38]. The transition region between the leaky-wave region and the guided mode region, where the mode propagates without attenuation, has also been studied [31, 34, 35, 39]. Lin et al. [34, 35] divided the radiation region into two subregions: the antenna mode region, where the greater part of the guided energy leaks transversely in the form of space and surface waves, and the reactive mode region, where most of the energy is reflected back to the feed line. Additionally, Lin et al. $[34,35]$ described a transition point from the reactive mode region to the antenna mode region, in which the real part (phase constant) and imaginary part (attenuation constant) of the complex propagation constant are equal to each other. In subsequent studies, Kim et al. [36, 41] showed that there were multiple antenna mode regions for a cylindrical dielectric rod, and Hirani et al. [37] presented these multiple antenna mode regions for a dielectric tube waveguide loaded with plasma.

Considering these studies, the mode regions for a cylindrical dielectric rod were classified as shown in Table 1, with the normalized propagation constant defined by Eq. (3).

In this study, TE, TM, and hybrid HE leaky modes for a cylindrical dielectric rod were investigated when the radius of the rod, $a$, was $10 \mathrm{~mm}$ and the relative dielectric constant, $\varepsilon_{n}$, was 4 . We used the guided modes for the structure used by Kim [32] and the leaky TE and TM modes for $a=5 \mathrm{~mm}$ and $\varepsilon_{r}=5[32,36,41]$. In our study, the TE and TM leaky modes were obtained and compared to the results available in the literature to check the accuracy of our results. As an original contribution, the hybrid HE modes were obtained using the coefficient matrix of the system of characteristic equations of the structure and Davidenko's method. The leaky-wave characteristics of the TE modes of the cylindrical dielectric rod when the radius of the dielectric rod was $10 \mathrm{~mm}$ and the relative dielectric constant was 4 are shown in Fig. 1.

Table 2 gives the spectral ranges of the TE modes for the structure.

The cutoff frequencies of the guided modes and their dispersion curves for the first three modes of $\mathrm{TE}_{0 \mathrm{n}}(\mathrm{n}=1,2$, and 3) have been presented by Kim [32]. The guided mode cutoff values and characteristics of the guided modes obtained in the current study were the same as the values obtained in the relevant research. The leaky-wave modes for the structure were also obtained in the current study. The spectral ranges of the leaky-wave region were $1.52,7.62,15.13$, and $22.97 \mathrm{GHz}$ for $\mathrm{TE}_{01}, \mathrm{TE}_{02}$, $\mathrm{TE}_{03}$, and $\mathrm{TE}_{04}$, respectively. The nonphysical mode region, where the normalized phase constant exceeded unity and the normalized attenuation constant was greater than zero, was physically meaningless $[31,36]$. In the nonphysical region, the normalized phase constant and the normalized attenuation constant increased rapidly, while the frequency decreased. As the frequency

Table 1. Mode region conditions

\begin{tabular}{lcc}
\hline Mode specifications & $\begin{array}{c}\text { Normalized } \\
\text { phase constant }\end{array}$ & $\begin{array}{c}\text { Normalized } \\
\text { attenuation constant }\end{array}$ \\
\hline $\begin{array}{l}\text { Nonphysical mode } \\
\text { Reactive mode }\end{array}$ & $\bar{\beta}>1$ & Large \\
Antenna mode & $\bar{\beta}<1$ & $\bar{\alpha}>1$ \\
Spectral gap & \multicolumn{1}{c}{$1>\bar{\beta} \geq \bar{\alpha}$} & \\
Guided mode & $\bar{\beta}>1$ & Small \\
\hline
\end{tabular}




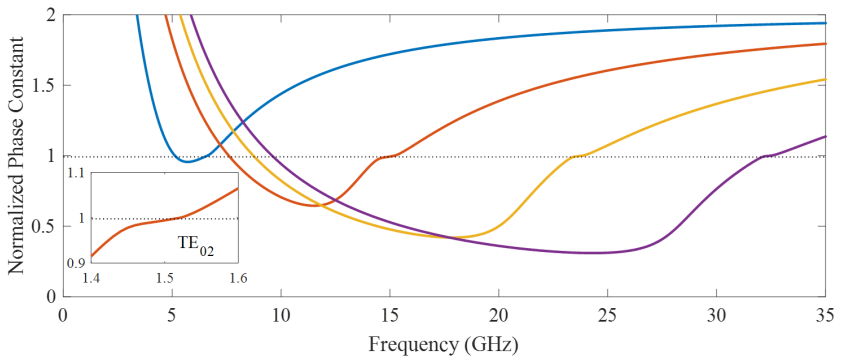

(a)

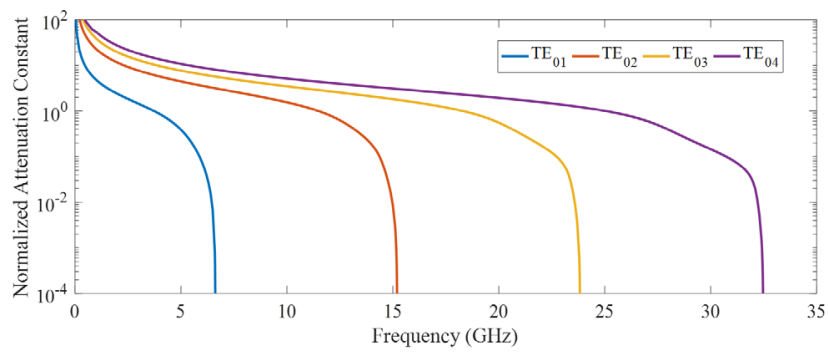

(b)

Fig. 1. Leaky-wave characteristics of the TE modes of the $10-\mathrm{mm}$ radius cylindrical dielectric rod with a relative dielectric constant of 4. (a) Normalized phase constant and (b) normalized attenuation constant.

Table 2. Spectral ranges of the TE modes for the $10-\mathrm{mm}$ radius cylindrical dielectric rod with a relative dielectric constant of 4

\begin{tabular}{ccccc}
\hline \multirow{2}{*}{ Mode } & Nonphysical & \multicolumn{2}{c}{ Leaky-wave region } & Guided mode \\
\cline { 3 - 4 } & mode & Reactive mode & Antenna mode & cutoff \\
\hline $\mathrm{TE}_{01}$ & $0-5.11$ & NA & $5.11-6.63$ & 6.63 \\
$\mathrm{TE}_{02}$ & $0-7.60$ & $7.60-12.38$ & $12.38-15.22$ & 15.22 \\
$\mathrm{TE}_{03}$ & $0-8.73$ & $8.73-20.15$ & $20.15-23.86$ & 23.86 \\
$\mathrm{TE}_{04}$ & $0-9.57$ & $9.57-27.74$ & $27.74-32.54$ & 32.54 \\
\hline
\end{tabular}

$\mathrm{NA}=$ not available.

increased outside this region, the reactive mode region first existed for the higher-order $\mathrm{TE}_{0 \mathrm{n}}(\mathrm{n} \geq 2)$ modes, and the antenna mode region existed at higher frequencies. The reactive mode region for the first order $\mathrm{TE}_{01}$ mode did not exist in the leaky-wave region, and the antenna mode region only existed for $\mathrm{TE}_{01}$. Also, the spectral gap did not exist in the TE modes, consistent with [32, $36,41]$. The spectral ranges of the antenna modes were obtained from Table 2 as $1.52,2.84,3.71$, and $4.8 \mathrm{GHz}$ for $\mathrm{TE}_{01}, \mathrm{TE}_{02}$,
$\mathrm{TE}_{03}$, and $\mathrm{TE}_{04}$, respectively. As seen from the results, the spectral width of the antenna mode region increased as the mode order increased. The normalized attenuation constant arrived at zero at the cutoff, and the guided modes existed above the cutoff frequency. In this region, the mode propagated unattenuated along the dielectric rod. The transition characteristic from the antenna mode region to the guided mode region for $\mathrm{TE}_{02}$ is given in the inset in Fig. 1(a).

The leaky-wave characteristics of the TM modes of the cylindrical dielectric rod are given in Fig. 2, where the radius of the dielectric rod was $10 \mathrm{~mm}$ and the relative dielectric constant was 4 . Table 3 gives the spectral ranges of the TM modes of the structure.

While the cutoff frequencies for the TM guided modes were the same as the cutoff frequencies for the TE guided modes and their guided mode characteristics were similar, the leaky-wave characteristics under the cutoff were substantially different. The spectral ranges of the leaky-wave region were 4.32, 13.97, 22.63, and

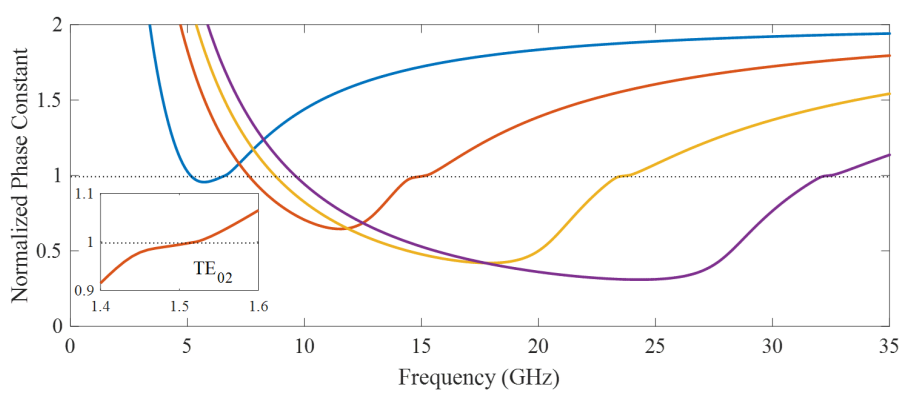

(a)

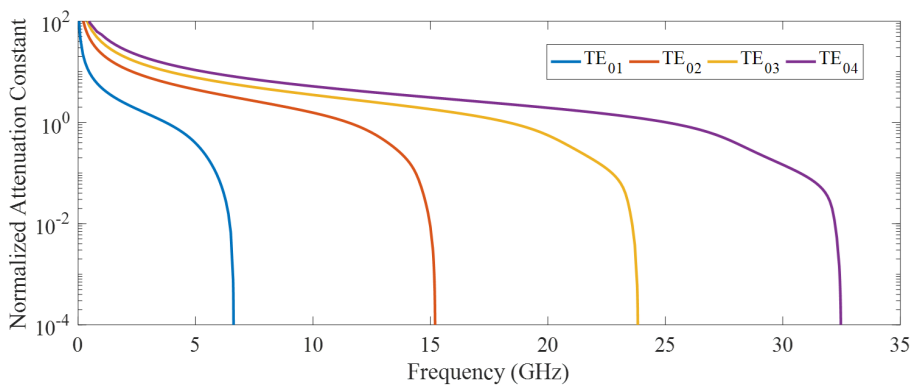

(b)

Fig. 2. Leaky-wave characteristics of the TM modes for the 10$\mathrm{mm}$ radius cylindrical dielectric rod with a relative dielectric constant 4. (a) Normalized phase constant and (b) normalized attenuation constant.

Table 3. Spectral ranges of the TM modes for the 10-mm radius cylindrical dielectric rod with a relative dielectric constant of 4

\begin{tabular}{|c|c|c|c|c|c|c|}
\hline \multirow{2}{*}{ Mode } & \multirow{2}{*}{$\begin{array}{c}\text { Nonphysical } \\
\text { mode }\end{array}$} & \multicolumn{4}{|c|}{ Leaky-wave region } & \multirow{2}{*}{$\begin{array}{l}\text { Guided mode } \\
\text { cutoff }\end{array}$} \\
\hline & & Reactive mode & 1st antenna mode & Spectral gap & 2nd antenna mode & \\
\hline $\mathrm{TM}_{01}$ & $0-2.31$ & NA & NA & NA & $2.31-6.63$ & 6.63 \\
\hline $\mathrm{TM}_{02}$ & $0-1.25$ & $1.25-9.59$ & $9.59-11.88$ & $11.88-13.50$ & $13.50-15.22$ & 15.22 \\
\hline $\mathrm{TM}_{03}$ & $0-1.23$ & $1.23-17.10$ & $17.10-20.89$ & $20.89-22.80$ & $22.80-23.86$ & 23.86 \\
\hline $\mathrm{TM}_{04}$ & $0-1.22$ & $1.22-24.55$ & $24.55-29.87$ & $29.87-31.74$ & $31.74-32.47$ & 32.47 \\
\hline
\end{tabular}

$\mathrm{NA}=$ not available. 
31.25 GHz for $\mathrm{TM}_{01}, \mathrm{TM}_{02}, \mathrm{TM}_{03}$, and $\mathrm{TM}_{04}$, respectively. The higher-order $\mathrm{TM}_{0 \mathrm{n}}$ modes $(\mathrm{n} \geq 2)$ divided the leaky-wave region into four subregions: reactive mode, 1 st antenna mode, spectral gap, and 2nd antenna mode. In addition, the nonphysical mode region was observed in the narrow spectrum for the TM modes than nonphysical mode region of $\mathrm{TE}$ modes. The $\mathrm{TM}_{01}$ mode only had one antenna mode distinct from the higher-order modes because the normalized phase constant value from the reactive mode region to the guided mode region was always greater than the normalized attenuation constant. For the higher-order modes $(\mathrm{n} \geq 2)$, the reactive mode region first existed above the nonphysical mode region. At higher frequencies, this was the 1 st antenna region, where the normalized attenuation constant was smaller than the normalized phase constant. As the frequency increased, there was a physically meaningless spectral gap that had the same properties as the nonphysical mode region. In both regions, the normalized phase constant exceeded unity, whereas the normalized attenuation constant differed from zero. In the frequency region above this physically meaningless region, the 2nd antenna mode region existed for the higher-order TM modes. The spectral ranges of the 1st antenna mode were 2.29, 3.79 , and $5.32 \mathrm{GHz}$ for $\mathrm{TM}_{02}, \mathrm{TM}_{03}$, and $\mathrm{TM}_{04}$, respectively. The spectral ranges of the 2nd antenna mode were 4.32, 1.72, 1.06, and $0.73 \mathrm{GHz}$ for $\mathrm{TM}_{01}, \mathrm{TM}_{02}, \mathrm{TM}_{03}$, and $\mathrm{TM}_{04}$, respectively. As seen from the results, the spectral width of the 1st antenna mode region was enlarged, and the spectral width of the 2 nd antenna mode narrowed as the mode order increased. An enlarged transition region from the 1st antenna mode region to the spectral gap and the 2nd antenna mode region and the guided mode region for $\mathrm{TM}_{02}$ and $\mathrm{TM}_{04}$ are presented in the insets in Fig. 2(a). The results obtained for the structure were consistent with the results obtained for the cylindrical dielectric rod with a radius of 5 $\mathrm{mm}$ and a relative dielectric constant of 5 in $[32,36,41]$.

As original research, the leaky-wave characteristics of the hybrid HE modes of the structure were obtained using Davidenko's method and the coefficient matrix of the system of characteristic equations described in the previous section. The leaky-wave characteristics of the hybrid HE modes are given in Fig. 3, where the radius of the dielectric rod was $10 \mathrm{~mm}$, the relative dielectric constant was 4 , and the azimuthal variation was $1(\mathrm{~m}=1)$. Table 4 presents

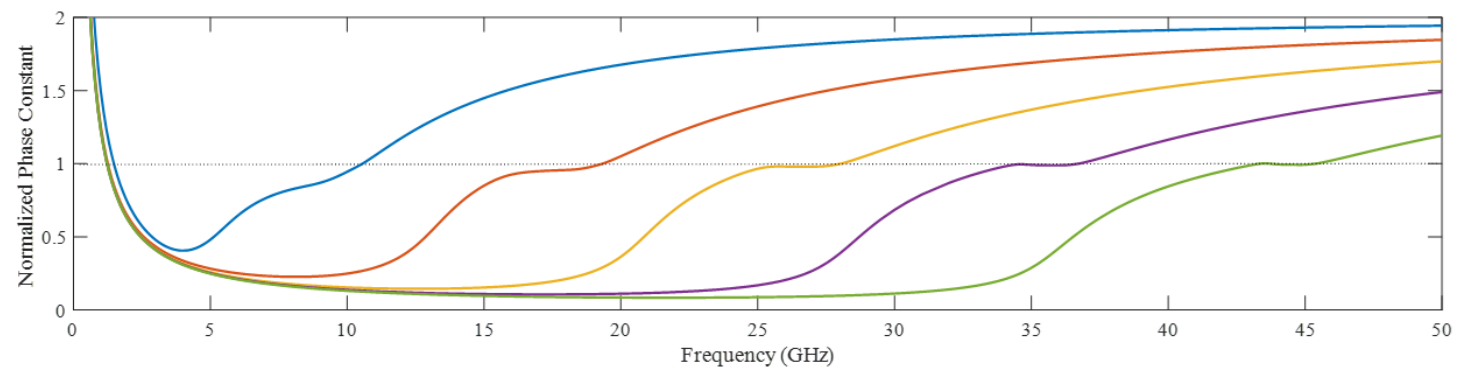

(a)

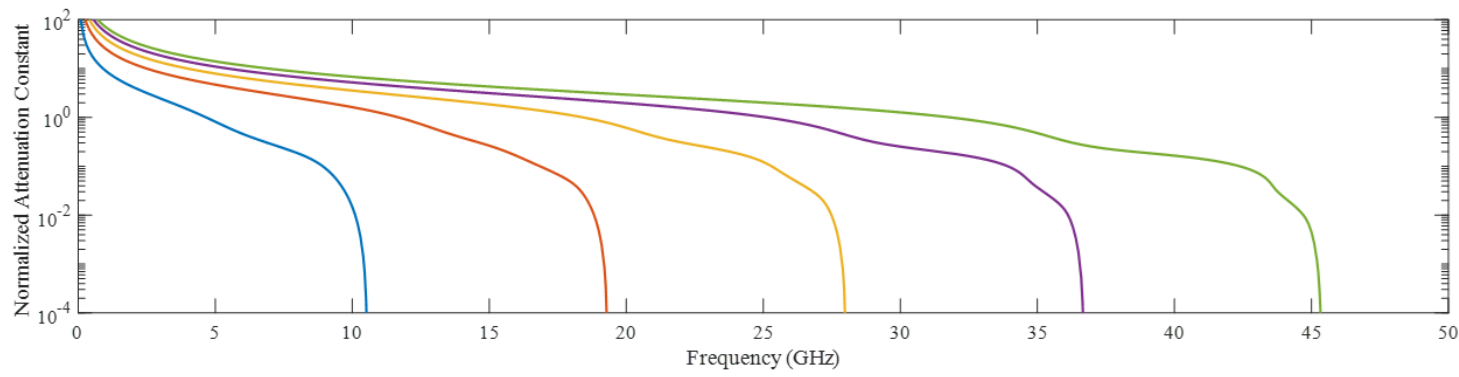

(b)

Fig. 3. Leaky-wave characteristics of the hybrid HE modes for the $10-\mathrm{mm}$ radius cylindrical dielectric rod with a relative dielectric constant of 4. (a) Normalized phase constant and (b) normalized attenuation constant.

Table 4. Spectral ranges of the hybrid HE modes for the $10-\mathrm{mm}$ radius cylindrical dielectric rod with a relative dielectric constant of 4

\begin{tabular}{|c|c|c|c|c|c|c|}
\hline \multirow{2}{*}{ Mode } & \multirow{2}{*}{$\begin{array}{c}\text { Nonphysical } \\
\text { mode }\end{array}$} & \multicolumn{4}{|c|}{ Leaky-wave region } & \multirow{2}{*}{$\begin{array}{l}\text { Guided mode } \\
\text { cutoff }\end{array}$} \\
\hline & & Reactive mode & 1st antenna mode & Spectral gap & 2nd antenna mode & \\
\hline $\mathrm{HE}_{11}$ & $0-1.47$ & $1.47-5.60$ & NA & NA & $5.60-10.57$ & 10.57 \\
\hline $\mathrm{HE}_{12}$ & $0-1.26$ & $1.26-13.14$ & NA & NA & $13.14-19.34$ & 19.34 \\
\hline $\mathrm{HE}_{13}$ & $0-1.24$ & $1.24-20.68$ & NA & NA & $20.68-28.05$ & 28.05 \\
\hline $\mathrm{HE}_{14}$ & $0-1.23$ & $1.23-28.19$ & NA & NA & $28.19-36.76$ & 36.76 \\
\hline $\mathrm{HE}_{15}$ & $0-1.22$ & $1.22-35.68$ & $35.68-43.34$ & $43.34-43.75$ & $43.75-45.37$ & 45.37 \\
\hline
\end{tabular}

$\mathrm{NA}=$ not available. 
the spectral ranges of the hybrid HE modes of the structure.

An open cylindrical guide structure supported the presence of the HEM modes and the TE and TM modes because all the field components could coexist both inside and outside the boundary due to the nonconducting boundary conditions. The coefficient matrix of the system of characteristic equations for the cylindrical dielectric rod and its derivation with respect to the propagation constant required by Davidenko's method are given in the appendix. The dispersion characteristics of the hybrid HE modes differed for both the TE and TM modes. To show these different characteristics, because the spectral gap was revealed after the fifth-order hybrid HE mode, the first five modes of the hybrid HE modes are presented in Fig. 3. The nonphysical mode regions existed within a narrow spectrum. In the leaky-wave region, the reactive mode region and the 2nd antenna mode region existed for all the HE modes, but the 1st antenna mode region and the spectral gap existed for the fifth-order $\left(\mathrm{HE}_{15}\right)$ and upper modes, as seen in Fig. 3 and Table 4. The behavior of the normalized phase constant determined the characteristic in the leakywave region, as shown in Fig. 4, an enlarged view of the normalized phase constant for the HE modes. For the higher hybrid modes ( $\mathrm{n} \geq 3$ ), the normalized phase constant increased until a certain frequency, later decreased, and then increased again while the frequency was rising. Although the behavior was similar, only $\mathrm{HE}_{15}$ in the first five order modes exceeded unity, and the spectral gap occurred. Because, spectral gap occurs while the phase constant higher than unity and the attenuation constant higher than zero. The spectral ranges of the 2nd antenna mode were 4.97, 6.2, 7.37, 8.57, and $1.62 \mathrm{GHz}$ for $\mathrm{HE}_{11}, \mathrm{HE}_{12}, \mathrm{HE}_{13}, \mathrm{HE}_{14}$, and $\mathrm{HE}_{15}$, respectively.

As seen from the listed results above, the spectral width of the 2nd antenna mode region was enlarged for the first four order modes when the mode order increased. $\mathrm{HE}_{15}$ had the narrowest 2nd antenna mode range, but it had two antenna mode regions. The spectral ranges of the leaky-wave region were 9.1, 18.08, 26.81, 35.53, and $44.15 \mathrm{GHz}$ for $\mathrm{HE}_{11}, \mathrm{HE}_{12}, \mathrm{HE}_{13}, \mathrm{HE}_{14}$, and $\mathrm{HE}_{15}$, respectively. The results show that the $\mathrm{HE}$ modes had a larger leaky-wave spectrum, particularly the antenna mode regions, compared to the TE and TM modes. This demonstrates that hybrid HE modes have a larger frequency spectrum for antenna applications because the antenna mode region mostly leaks power into the space wave.

\section{CONCLUSION}

The leaky-wave characteristics of TE and TM modes for a cylindrical dielectric rod in the literature were obtained from the joint application of the dispersion equation of the structure and Davidenko's method. Davidenko's method requires the derivative of the dispersion equation with respect to the propagation constant. It can be hard to obtain these derivations, especially for complex expressions. In this study, which addressed a gap in the literature, the coefficient matrix of the system of characteristic equations for the structure was used instead of the dispersion relation so that the derivative expression required by Davidenko's method could thereby be readily obtained. In so doing, a complete set of analytical solutions was obtained without the need for currently employed auxiliary software programs and derivative processes. This approximation enables Davidenko's method to be used for complex structures, such as an anisotropic medium, due to more complicated dispersion equations. In this study, the spectral ranges of a nonphysical mode, a 1st antenna mode, a spectral

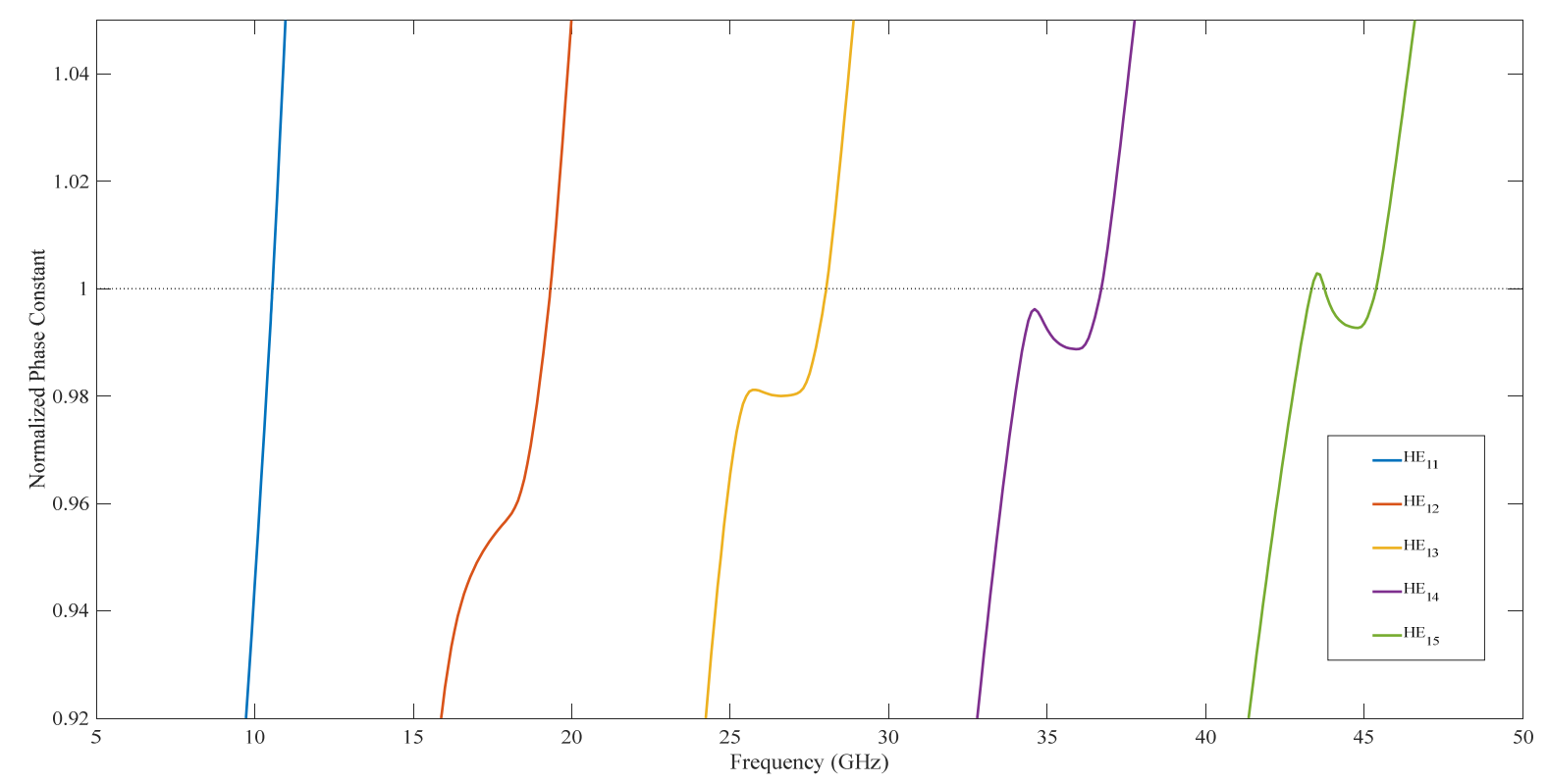

Fig. 4. Enlarged view of the normalized phase constants of the hybrid HE modes in the vicinity of unity. 
gap, a 2nd antenna mode, and guided modes were obtained for both the TE and TM modes of a structure with a $10-\mathrm{mm}$ radius and a relative dielectric constant of 4 . The results are compatible with the results in the literature, which show that $\mathrm{TE}$ modes have only one antenna mode region, while TM modes $(n \geq 2)$ have two antenna mode regions. Additionally, the 1st antenna mode region, the spectral gap, and the 2 nd antenna mode region exist for higher-order TM modes $(\mathrm{n} \geq 2)$.

The cylindrical dielectric rod supports the presence of HEM modes and TE and TM modes because all magnetic and electric field components exist both inside and outside the boundary. In this study, the HEM modes for the cylindrical dielectric rod were obtained, which represents an original contribution to the literature. Using the coefficient matrix of the system of characteristic equations instead of the dispersion equation enabled the HEM modes to be obtained because the derivative expression required by Davidenko's method could be readily found. The first five order hybrid HE modes were obtained and presented because the spectral gap was revealed after the fifth-order HE mode. The first four $\mathrm{HE}$ modes had both reactive mode and 2 nd antenna mode regions within the leaky-wave region. However, for the fifthorder HE mode and upper modes, the reactive mode, the 1st antenna mode, the spectral gap, and the 2nd antenna mode existed in the leaky-wave region. Additionally, the HE modes had a wider leaky-wave spectrum and antenna mode regions than the TE and TM modes. This indicates that HE modes have a larger frequency spectrum for antenna applications because the antenna mode region leaks are significantly radiated into the space wave.

\section{REFERENCES}

[1] S. S. Yi, "Observation of the scattered TE- and TM-mode coupling in active dielectric cylinder," Optics Communications, vol. 173, no. 1-6, pp. 211-215, 2000.

[2] M. Moisan, A. Shivarova, and A. W. Trivelpiece, "Experimental investigations of the propagation of surface waves along a plasma column," Plasma Physics, vol. 24, no. 11, pp. 1331-1400, 1982.

[3] H. Haus and D. Miller, "Attenuation of cutoff modes and leaky modes of dielectric slab structures," IEEE Journal of Quantum Electronics, vol. 22, no. 2, pp. 310-318, 1986.

[4] Y. Yu and L. Cao, "Leaky mode engineering: a general design principle for dielectric optical antenna solar absorbers," Optics Communications, vol. 314, pp. 79-85, 2014.

[5] F. Xu and K. Wu, "Understanding leaky-wave structures: a special form of guided-wave structure," IEEE Microwave Magazine, vol. 14, no. 5, pp. 87-96, 2013.

[6] D. R. Jackson, P. Burghignoli, G. Lovat, F. Capolino, J. Chen, D. R. Wilton, and A. A. Oliner, "The fundamental physics of directive beaming at microwave and optical fre- quencies and the role of leaky waves," Proceedings of the IEEE, vol. 99, no. 10, pp. 1780-1805, 2011.

[7] V. Ebrahimi, L. Yousefi, and M. Mohammad-Taheri, "Enabling inter- and intra-chip optical wireless interconnect by the aid of hybrid plasmonic leaky-wave optical antennas," Optics Communications, vol. 382, pp. 119-126, 2017.

[8] N. Hussain, K. E. Kedze, and I. Park, "Performance of a planar leaky-wave slit antenna for different values of substrate thickness," Journal of Electromagnetic Engineering and Science, vol. 17, no. 4, pp. 202-207, 2017.

[9] P. Burghignoli, W. Fuscaldo, D. Comite, P. Baccarelli, and A. Galli, "Higher-order cylindrical leaky waves-Part I: Canonical sources and radiation formulas," IEEE Transactions on Antennas and Propagation, vol. 67, no. 11, pp. 67356747, 2019.

[10] F. Monticone and A. Alu, "Leaky-wave theory, techniques, and applications: from microwaves to visible frequencies," Proceedings of the IEEE, vol. 103, no. 5, pp. 793-821, 2015.

[11] J. Wan, O. Rybin, and S. Shulga, "Far field focusing for a microwave patch antenna with composite substrate," $R e^{-}$ sults in Physics, vol. 8, pp. 971-976, 2018.

[12] G. Lu, F. M. Sabirova, and A. V. Morozov, "Simulation and experimental measurement of digital multi-beamforming phased antenna array in the frequency range C," Results in Physics, vol. 13, article no. 102310, 2019. https://doi. org/10.1016/j.rinp.2019.102310

[13] A. M. Tamim, M. R. I. Faruque, M. J. Alam, S. S. Islam, and M. T. Islam, "Split ring resonator loaded horizontally inverse double L-shaped metamaterial for $\mathrm{C}$-, $\mathrm{X}$-and $\mathrm{Ku}$ band microwave applications," Results in Physics, vol. 12, pp. 2112-2122, 2019.

[14] N. Berahim, I. S. Amiri, T. Anwar, S. R. Azzuhri, M. M. Nasir, R. Zakaria, et al., "Polarizing effect of MoSe2coated optical waveguides," Results in Physics, vol. 12, pp. 7-11, 2019.

[15] N. K. Mishra, S. Das, and D. K. Vishwakarma, "Beam steered linear array of cylindrical dielectric resonator antenna," AEU-International Journal of Electronics and Communications, vol. 98, pp. 106-113, 2019.

[16] A. Bostani and H. Oraizi, "Analysis and design of radially inhomogeneous tapered dielectric rod antennas," AEUInternational Journal of Electronics and Communications, vol. 101, pp. 106-113, 2019.

[17] S. T. Yang and H. Ling, "Application of compressive sensing to two-dimensional radar imaging using a frequencyscanned microstrip leaky wave antenna," Journal of Electromagnetic Engineering and Science, vol. 17, no. 3, pp. 113119, 2017.

[18] P. Ranjan and R. K. Gangwar, "Probe feed half split cylindrical dielectric resonator antenna for wideband appli- 
cation," AEU-International Journal of Electronics and Communications, vol. 69, no. 11, pp. 1709-1714, 2015.

[19] J. Zhu and J. Lou, "High-sensitivity Fano resonance temperature sensor in MIM waveguides coupled with a polydimethylsiloxane-sealed semi-square ring resonator," $R e-$ sults in Physics, vol. 18, article no. 103183, 2020. https:// doi.org/10.1016/j.rinp.2020.103183

[20] G. Das, N. K. Sahu, and R. K. Gangwar, "Dielectric resonator based multiport antenna system with multi-diversity and built-in decoupling mechanism," AEU-International Journal of Electronics and Communications, vol. 119, article no. 153193, 2020. https://doi.org/10.1016/j.aeue.2020.15 3193

[21] T. Ramachandran, M. R. I. Faruque, and M. T. Islam, "A dual band left-handed metamaterial-enabled design for satellite applications," Results in Physics, vol. 16, article no. 102942, 2020. https://doi.org/10.1016/j.rinp.2020.102942

[22] W. Su, Y. Liu, and B. Chen, "Multiple Fano resonances in asymmetric rectangular ring resonator based on graphene nanoribbon," Results in Physics, vol. 17, article no. 103121, 2020. https://doi.org/10.1016/j.rinp.2020.103121

[23] I. S. Amiri, T. Anwar, R. Zakaria, and P. Yupapin, "TElike mode analysis of microsystem $\mathrm{InGaAsP} / \mathrm{InP}$ semiconductor resonator generating $20 \mathrm{GHz}$ repetition rate pulse trains," Results in Physics, vol. 10, pp. 980-986, 2018.

[24] I. S. Amiri, F. Alizadeh, M. M. Ariannejad, R. Amini, and P. Yupapin, "Computation of ion exchange buried microring resonator waveguide for $\mathrm{THz}$ communication applications," Results in Physics, vol. 10, pp. 287-290, 2018.

[25] M. M. Ariannejad, I. S. Amiri, S. R. B. Azzuhri, R. Zakaria, and P. Yupapin, "Polarization dependence of SU-8 micro ring resonator," Results in Physics, vol. 11, pp. 515-522, 2018.

[26] X. Lu, G. G. Zheng, and P. Zhou, "High performance refractive index sensor with stacked two-layer resonant waveguide gratings," Results in Physics, vol. 12, pp. 759$765,2019$.

[27] B. Zeng, H. Xu, M. Zhao, C. Xiong, C. Liu, and H. Li, "Optical tunable modulator based on graphene metamaterials at terahertz communication range," Results in Physics, vol. 16, article no. 102935, 2020. https://doi.org/ 10.1016/j.rinp.2020.102935

[28] P. Wu, Z. Chen, H. Jile, C. Zhang, D. Xu, and L. Lv, "An infrared perfect absorber based on metal-dielectric-metal multi-layer films with nanocircle holes arrays," Results in Physics, vol. 16, article no. 102952, 2020. https://doi.org/ 10.1016/j.rinp.2020.102952

[29] J. Hu and C. R. Menyuk, "Understanding leaky modes: slab waveguide revisited," Advances in Optics and Photonics, vol. 1, no. 1, pp. 58-106, 2009.
[30] A. A. Oliner, "Leakage from higher modes on microstrip line with application to antennas," Radio Science, vol. 22, no. 6. pp. 907-912, 1987.

[31] P. Lampariello, F. Frezza, and A. A. Oliner, "The transition region between bound-wave and leaky-wave ranges for a partially dielectric-loaded open guiding structure," IEEE Transactions on Microwave Theory and Techniques, vol. 38, no. 12, pp. 1831-1836, 1990.

[32] K. Y. Kim, "Guided and leaky modes of circular open electromagnetic waveguides: dielectric, plasma, and metamaterial columns," Ph.D. dissertation, Kyungpook National University, Daegu, Korea, 2004.

[33] X. Y. Zeng, S. J. Xu, K. Wu, and K. M. Luk, "Properties of guided modes on open structures near the cutoff region using a new version of complex effective dielectric constant," IEEE Transactions on Microwave Theory and Techniques, vol. 50, no. 5, pp. 1417-1424, 2002.

[34] Y. D. Lin, J. W. Sheen, and C. C. Tzuang, "Analysis and design of feeding structures for microstrip leaky wave antenna," IEEE Transactions on Microwave Theory and Techniques, vol. 44, no. 9, pp. 1540-1547, 1996.

[35] Y. D. Lin and J. W. Sheen, "Mode distinction and radiation-efficiency analysis of planar leaky-wave line source," IEEE Transactions on Microwave Theory and Techniques, vol. 45, no. 10, pp. 1672-1680, 1997.

[36] K. Y. Kim, H. S. Tae, and J. H. Lee, "Leaky dispersion characteristics in circular dielectric rod using Davidenko's method," Journal of the Korea Electromagnetic Engineering Society, vol. 5, no. 2, pp. 72-79, 2005.

[37] R. R. Hirani, S. K. Pathak, S. N. Shah, and D. K. Sharma, "Dispersion characteristics of dielectric tube waveguide loaded with plasma for leaky wave antenna application," AEU-International Journal of Electronics and Communications, vol. 83, pp. 123-130, 2018.

[38] K. A. Michalski and D. Zheng, "Rigorous analysis of open microstrip lines of arbitrary cross-section in bound and leaky regimes," IEEE Transactions on Microwave Theory and Techniques, vol. 37, no. 12, pp. 2005-2010, 1989.

[39] J. S. Bagby, C. H. Lee, D. P. Nyquist, and Y. Yuan, "Identification of propagation regimes on integrated microstrip transmission lines," IEEE Transactions on Microwave Theory and Techniques, vol. 41, no. 11, pp. 1887-1894, 1993.

[40] C. Luxey and J. M. Laheurte, "Simple design of dualbeam leaky-wave antennas in microstrips," IEE Proceedings-Microwaves, Antennas and Propagation, vol. 144, no. 6, pp. 397-402, 1997.

[41] K. Y. Kim, H. S. Tae, and J. H. Lee, "Analysis of leaky modes in circular dielectric rod waveguides," Electronics Letters, vol. 39, no. 1, pp. 61-62, 2003.

[42] C. A. Balanis, Advanced Engineering Electromagnetics. Ho- 
boken, NJ: John Wiley \& Sons, 1989.

[43] C. Yeh and F. I. Shimabukuro, The Essence of Dielectric Waveguides. Boston, MA: Springer, 2008.

[44] C. Yeh, "Guided-wave modes in cylindrical optical fibers," IEEE Transactions on Education, vol. 30, no. 1, pp. 43-51, 1987.

[45] E. Snitzer, "Cylindrical dielectric waveguide modes," Journal of the Optical Society of America, vol. 51, no. 5, pp. 491-498, 1961.

[46] K. Morishita, "Hybrid modes in circular cylindrical optical fibers," IEEE Transactions on Microwave Theory and Tecbniques, vol. 31, no. 4, pp. 344-350, 1983.

[47] W. M. Bruno and W. B. Bridges, "Flexible dielectric waveguides with powder cores," IEEE Transactions on Microwave Theory and Techniques, vol. 36, no. 5, pp. 882890, 1988.

[48] H. Talisa, "Application of Davidenko's method to the solution of dispersion relations in lossy waveguiding systems," IEEE Transactions on Microwave Theory and Techniques, vol. 33, no. 10, pp. 967-971, 1985.

[49] H. A. N. Hejase, "On the use of Davidenko's method in complex root search," IEEE Transactions on Microwave Theory and Techniques, vol. 41, no. 1, pp. 141-143, 1993.

[50] K. Naishadham and L. B. Felsen, "Dispersion of waves guided along a cylindrical substrate-superstrate layered medium," IEEE Transactions on Antennas and Propagation, vol. 41, no. 3, pp. 304-313, 1993.

[51] K. Naishadham and H. W. Yao, "An efficient computation of transient scattering by a perfectly conducting cylinder,"

\section{Ersoy Kelebekler}

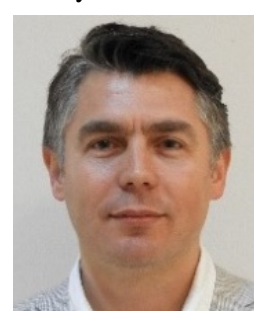

was born in Bursa, Turkey, in 1980. He received his B.E. degree in electronic technical education from Kocaeli University, Turkey in 2002. He also received his M.S. degree and Ph.D. in electronic and computer education from Kocaeli University in 2006 and 2012, respectively. He joined the Department of Electricity and Energy at Uzunciftlik Nuh Cimento VHS of Kocaeli University in 2013. His current research interests include theoretical electromagnetics, waveguides, electromagnetic compatibility in power electronics, low-power wireless sensor networks, and distributed measurement systems.
IEEE Transactions on Antennas and Propagation, vol. 41, no. 11, pp. 1509-1515, 1993.

[52] M. M. Shabat, "Numerical algorithms for modeling hybrid surface plasmon polaritons guided by metal films," Microwave and Optical Technology Letters, vol. 16, no. 2, pp. 122-124, 1997.

[53] M. M. Shabat, D. Jager, N. M. Barakat, G. Strip, and P. Authority, "Numerical and analytical solutions of dispersion equation in lossy nonlinear waveguiding system," Microwave and Optical Technology Letters, vol. 22, no. 4, pp. 273-278, 1999.

[54] M. M. Shabat, "Application of Davidenko's method to a lossy nonlinear waveguide," International Journal of Infrared and Millimeter Waves, vol. 20, no. 7, pp. 1363-1370, 1999.

[55] M. M. Shabat, Y. S. Madi, and D. Jager, "Solution of lossy dielectric moving waveguides using numerical method (Davidenko's approach)," Electromagnetics, vol. 22, no. 8, pp. 659-666, 2002.

[56] Y. Liu and T. Itoh, "Control of leakage in multilayered conductor-backed coplanar structures," in Proceedings of 1994 IEEE MTT-S International Microwave Symposium Digest (Cat. No. 94CH3389-4), San Diego, CA, 1994, pp. 141-144.

[57] D. Nghiem, J. T. Williams, D. R. Jackson, and A. A. Oliner, "Suppression of leakage on stripline and microstrip structures," in Proceedings of 1994 IEEE MTT-S International Microwave Symposium Digest (Cat. No. 94CH33894), San Diego, CA, 1994, pp. 145-148. 


\section{APPENDIX}

This section presents the coefficient matrix of the system of the characteristic equations for a cylindrical dielectric rod surrounded by free space when the medium is lossless and sourceless and the relative dielectric constant and the radius of the dielectric are $\varepsilon_{r}$ and $a$, respectively. The variation of the field is defined as $F(r, \varphi, z)=F(r) e^{j(\omega t-\gamma z-m \varphi)}$, where $\omega$ is the angular frequency, $\gamma$ is the complex propagation constant, and $m$ is the azimuthal variation. The system of the characteristic equations for the structure is obtained from Maxwell equations, and the boundary and continuity conditions between the dielectric rod and the free space, where the $z$ and $\varphi$ components of the electric and magnetic fields are equal to each other. The system of the characteristic equations for the cylindrical dielectric rod surrounded by free space is obtained from the Eq. (A.1).

In Eq. (A.1), $J_{\mathrm{m}}$ and $H_{m}^{(2)}$ are the first kind Bessel Function and the second kind Hankel Function, respectively. The apostrophe above the Bessel and Hankel functions indicates the derivative of the function; $\varepsilon_{0}$ and $\mu_{0}$ are the permittivity and permeability of the free space, respectively; and $j$ is the imaginary unit number. $k_{0}, k_{1}$, and $k_{2}$ are described as follows:

$$
\begin{aligned}
k_{0} & =\sqrt{\omega^{2} \varepsilon_{0} \mu_{0}} . \\
k_{1} & =\sqrt{k_{0}^{2} \varepsilon_{r}-\gamma^{2}} \\
k_{2} & =\sqrt{k_{0}^{2}-\gamma^{2}} .
\end{aligned}
$$

If the coefficient matrix of Eq. (A.1) is called $M$, its derivative with respect to the propagation constant is obtained by the derivative of each element of the matrix with respect to the corresponding variable.

$$
\frac{d \boldsymbol{M}}{d \gamma}=\left[\begin{array}{llll}
\frac{d M_{11}}{d \gamma} & \frac{d M_{12}}{d \gamma} & \frac{d M_{13}}{d \gamma} & \frac{d M_{14}}{d \gamma} \\
\frac{d M_{21}}{d \gamma} & \frac{d M_{22}}{d \gamma} & \frac{d M_{23}}{d \gamma} & \frac{d M_{24}}{d \gamma} \\
\frac{d M_{31}}{d \gamma} & \frac{d M_{32}}{d \gamma} & \frac{d M_{33}}{d \gamma} & \frac{d M_{34}}{d \gamma} \\
\frac{d M_{41}}{d \gamma} & \frac{d M_{42}}{d \gamma} & \frac{d M_{43}}{d \gamma} & \frac{d M_{44}}{d \gamma}
\end{array}\right]
$$

where

$$
\begin{gathered}
\frac{d M_{21}}{d \gamma}=\frac{d M_{23}}{d \gamma}=\frac{d M_{42}}{d \gamma}=\frac{d M_{44}}{d \gamma}=0 \\
\frac{d M_{22}}{d \gamma}=\frac{d M_{41}}{d \gamma}=-\frac{\gamma a}{k_{1}} J_{m}^{\prime}\left(k_{1} a\right) \\
\frac{d M_{24}}{d \gamma}=\frac{d M_{43}}{d \gamma}=\frac{\gamma a}{k_{2}} H_{m}^{\prime(2)}\left(k_{2} a\right) \\
\frac{d M_{11}}{d \gamma}=\frac{d M_{32}}{d \gamma}=\frac{m \gamma}{a k_{1}^{3}}\left[-\frac{k_{1}^{2}+2 \gamma^{2}}{\gamma k_{1}} J_{m}\left(k_{1} a\right)+\gamma a J_{m}^{\prime}\left(k_{1} a\right)\right] \\
\frac{d M_{12}}{d \gamma}=-j \frac{k_{0}^{2} \gamma}{\omega \varepsilon_{0} k_{1}}\left[-\frac{1}{k_{1}^{2}} J_{m-1}\left(k_{1} a\right)+\frac{2 m}{k_{1}^{3} a} J_{m}\left(k_{1} a\right)\right. \\
\left.+\frac{a}{k_{1}} J_{m-1}^{\prime}\left(k_{1} a\right)-\frac{m}{k_{1}^{2}} J_{m}^{\prime}\left(k_{1} a\right)\right]
\end{gathered}
$$

$\frac{d M_{13}}{d \gamma}=\frac{d M_{34}}{d \gamma}=-\frac{m \gamma}{a k_{2}^{3}}\left[-\frac{k_{2}^{2}+2 \gamma^{2}}{\gamma k_{2}} H_{m}^{(2)}\left(k_{2} a\right)+\gamma a H_{m}^{\prime(2)}\left(k_{2} a\right)\right]$

$$
\begin{aligned}
\frac{d M_{14}}{d \gamma}= & j \frac{k_{0}^{2} \gamma}{\omega \varepsilon_{0} k_{2}}\left[-\frac{1}{k_{2}^{2}} H_{m-1}^{(2)}\left(k_{2} a\right)+\frac{2 m}{k_{2}^{3} a} H_{m}^{(2)}\left(k_{2} a\right)\right. \\
& \left.+\frac{a}{k_{2}} H_{m-1}^{\prime(2)}\left(k_{2} a\right)-\frac{m}{k_{2}^{2}} H_{m}^{(2)}\left(k_{2} a\right)\right]
\end{aligned}
$$

$$
\frac{d M_{31}}{d \gamma}=j \frac{k_{0}^{2} \varepsilon_{r} \gamma}{\omega \mu_{0} k_{1}}\left[-\frac{1}{k_{1}^{2}} J_{m-1}\left(k_{1} a\right)+\frac{2 m}{k_{1}^{3} a} J_{m}\left(k_{1} a\right)\right.
$$$$
\left.+\frac{2 m}{k_{1}^{3} a} J_{m}\left(k_{1} a\right)+\frac{a}{k_{1}} J_{m-1}^{\prime}\left(k_{1} a\right)-\frac{m}{k_{1}^{2}} J_{m}^{\prime}\left(k_{1} a\right)\right]
$$$$
\frac{d M_{33}}{d \gamma}=-j \frac{k_{0}^{2} \gamma}{\omega \mu_{0} k_{2}}\left[-\frac{1}{k_{2}^{2}} H_{m-1}^{(2)}\left(k_{2} a\right)+\frac{2 m}{k_{2}^{3} a} H_{m}^{(2)}\left(k_{2} a\right)\right.
$$$$
\left.+\frac{a}{k_{2}} H_{m-1}^{(2)}\left(k_{2} a\right)-\frac{m}{k_{2}^{2}} H_{m}^{\prime(2)}\left(k_{2} a\right)\right]
$$

$$
\left[\begin{array}{cccc}
-\frac{m}{a} \frac{\gamma}{k_{1}^{2}} J_{m}\left(k_{1} a\right) & j \frac{1}{\omega \varepsilon_{0}} \frac{k_{0}^{2}}{k_{1}} J_{m}^{\prime}\left(k_{1} a\right) & \frac{m}{a} \frac{\gamma}{k_{2}^{2}} H_{m}^{(2)}\left(k_{2} a\right) & -j \frac{1}{\omega \varepsilon_{0}} \frac{k_{0}^{2}}{k_{2}} H_{m}^{\prime(2)}\left(k_{2} a\right) \\
0 & J_{m}\left(k_{1} a\right) & 0 & -H_{m}^{(2)}\left(k_{2} a\right) \\
-j \frac{1}{\omega \mu_{0}} \frac{k_{0}^{2} \varepsilon_{1}}{k_{1}} J_{m}^{\prime}\left(k_{1} a\right) & -\frac{m}{a} \frac{\gamma}{k_{1}^{2}} J_{m}\left(k_{1} a\right) & j \frac{1}{\omega \mu_{0}} \frac{k_{0}^{2}}{k_{2}} H_{m}^{(2)}\left(k_{2} a\right) & \frac{m}{a} \frac{\gamma}{k_{2}^{2}} H_{m}^{(2)}\left(k_{2} a\right) \\
J_{m}\left(k_{1} a\right) & 0 & -H_{m}^{(2)}\left(k_{2} a\right) & 0
\end{array}\right]\left[\begin{array}{l}
A \\
B \\
C \\
D
\end{array}\right]=0
$$

